\title{
Dwarf galaxies are among the best places to probe the nature of dark matter
}

\section{S. Colafrancesco}

INAF - Osservatorio Astronomico di Roma, Via Frascati 33, Monteporzio, I00040, Italy. email: cola@mporzio.astro.it

\begin{abstract}
I discuss here the main physical constraints on Dark Matter particles that can be obtained by the study of the astrophysical signals produced by their annihilation within Dwarf Galaxies. The present results are obtained in the framework of neutralino Dark Matter scenarios and under the assumption that Dwarf Galaxies are dominated by Dark Matter.
\end{abstract}

Keywords. Dwarf galaxies, Dark Matter, Cosmology

\section{Signals from the Dark Universe}

Dark Matter (DM) is everywhere in the universe. This is a lessons we learned from cosmology and from models of structure formation. The first teacher who pointed out the existence of DM in large-scale structures was F.Zwicky (1933) who realized that the Coma cluster of galaxies is dominated by DM from the study of the galactic dynamics. The evidence for the presence of DM in systems of galaxies accumulated since Zwicky's age, and we know today that dark halos are found around all galaxy systems, from galaxy clusters to giant ellipticals and to dwarf galaxies.

The lesson we received from observational cosmology (WMAP vs. distant SN Ia) indicated that the matter content of the universe is $\Omega_{m} h^{2}=0.135_{-0.009}^{+0.008}$ with a baryon density of $\Omega_{b} h^{2}=0.0224 \pm 0.0009$ (Spergel et al. (2003)). These values translate to matter density parameters $\Omega_{m} \approx 0.27 \pm 0.04$ and $\Omega_{b} \approx 0.044 \pm 0.004$ for the value $h=0.71_{-0.03}^{+0.04}$ (Page (2003)). This means that the amount of material which is in the form of a Dark non-baryonic Matter should be $\Omega_{D M} \approx 0.23$ (range $0.21-0.25$ ) assuming $h=0.7$.

Although we know that DM is the most abundant and common form of matter in the universe, we do not know yet what its nature. Dark Matter has to be in the form of cold particles in order to reproduce the observed features of the large-scale distribution of matter in the universe. Among the viable competitors for having a cosmologically relevant DM species, the leading candidate is the lightest supersymmetric (SUSY) particle, plausibly the neutralino $\chi$, with a mass $M_{\chi}$ in the range between a few $\mathrm{GeV}$ to a few hundreds of GeV (Jungman et al. (1996)).

The dark side of the universe sends us signals of the presence and of the nature of its constituents that can be recorded using different astrophysical probes. These probes are of inference and physical character.

Inference probes of DM (e.g., the CMB anisotropy spectrum, the dynamics of galaxies, the hydrodynamics of the hot intra-cluster gas and the gravitational lensing distortion of background galaxies by the intervening potential wells of galaxy clusters) can be used to assess the presence, the amount and the spatial distribution of DM in large scale structures but are not able to provide information on its nature.

Physical probes tell us about the nature and the physical properties of the DM particles and come thru the analysis of the astrophysical effects of DM interaction, which can be directly or indirectly observed by astrophysical techniques. These signals involve, in 
the case of a neutralino $(\chi)$ DM, emission of gamma-rays, neutrinos, synchrotron and bremsstrahlung radiation together with the Inverse Compton Scattering (ICS) of the CMB (and other background) photons and the SZ effect produced by the secondary electrons produced in the DM annihilation process (see Fig.1 and Colafrancesco (2003), Colafrancesco (2004a), Colafrancesco (2005b) for details).

\section{Dark Matter signals from Dwarf Galaxies}

The best places to probe DM nature in the universe are galaxy clusters and dwarf galaxies: the former are the largest bound containers of DM in the universe while the latter are the smaller and closer approximation to a pure DM halo. We will discuss here the case of dwarf galaxies, while the case of galaxy clusters has been discussed elsewhere (e.g., Colafrancesco \& Mele (2001), Colafrancesco (2003), 2004b).

Dwarf galaxies, in fact, seem to be dominated by DM both in their central regions and in their outskirts (see, e.g., W.Ewans, M.Mateo, at this Meeting) and many of them have undergone an efficient star formation which consumed most of their gas content. Moreover, these systems contain little or no hot gas, as shown by absence of X-ray emission, and a lack of relativistic particles (cosmic rays), as shown by the absence of radio emission. Furthermore, we have evidence of very close dwarf galaxies (a dozen of which are in the MW environment or around nearby galaxies like M31) which hence allow us to study in details the astrophysical features of the DM annihilation signals. Here we consider specifically the case of the Draco dwarf galaxy, whose structural and physical properties have been discussed extensively at this Meeting.

\subsection{DM annihilation in dwarf galaxies}

The $\chi$ annihilation rate in a DM halo is $R=n_{\chi}(r)\langle\sigma V\rangle_{A}$, where $n_{\chi}(r)=n_{\chi, 0} g(r)$ is the neutralino number density and $\langle\sigma V\rangle_{A}$ is the $\chi \chi$ annihilation cross section averaged over a thermal velocity distribution at freeze-out temperature (Jungman et al. (1996)). Here we assume a cosmologically relevant DM with a cuspy DM density profile $g(r)=\left(r / r_{s}\right)^{-1}(1+$ $\left.r / r_{s}\right)^{-2}$ (e.g., Navarro, Frenk \& White (1997)) consistent with both numerical simulations and with velocity dispersion profiles. Although the $\chi \chi$ annihilation cross section is a nontrivial function of the mass and physical composition of the neutralino, to our purpose it suffices to recall that the $\chi$ relic density is approximately given by

$$
\Omega_{\chi} h^{2} \simeq \frac{3 \times 10^{-27} \mathrm{~cm}^{3} \mathrm{~s}^{-1}}{\langle\sigma V\rangle_{A}} .
$$

Enhancing (suppressing) the $\chi$ annihilation rate will have the effect of rescaling the final secondary products $\left(e^{ \pm}, \pi^{0, \pm}\right)$ spectra by the same enhancement (suppression) factor. Neutralinos which annihilate inside a DM halo produce quarks, leptons, vector bosons and Higgs bosons, depending on their mass and physical composition. Electrons are then produced from the decay of the final heavy fermions and bosons (monochromatic electrons, with energy about $M_{\chi}$, coming from the direct channel $\chi \chi \rightarrow e e$, are in general much suppressed). The different composition of the $\chi \chi$ annihilation final state will in general affect the form of the final electron spectrum. We use here DM source spectra which are obtained from Monte Carlo simulations (e.g., DarkSUSY, Gondolo et al. (2004)). Analytical, approximated expressions for the $\mathrm{e}^{ \pm}$spectrum which resume the relevant aspects of detailed numerical studies has been given by Colafrancesco \& Mele (2001). 


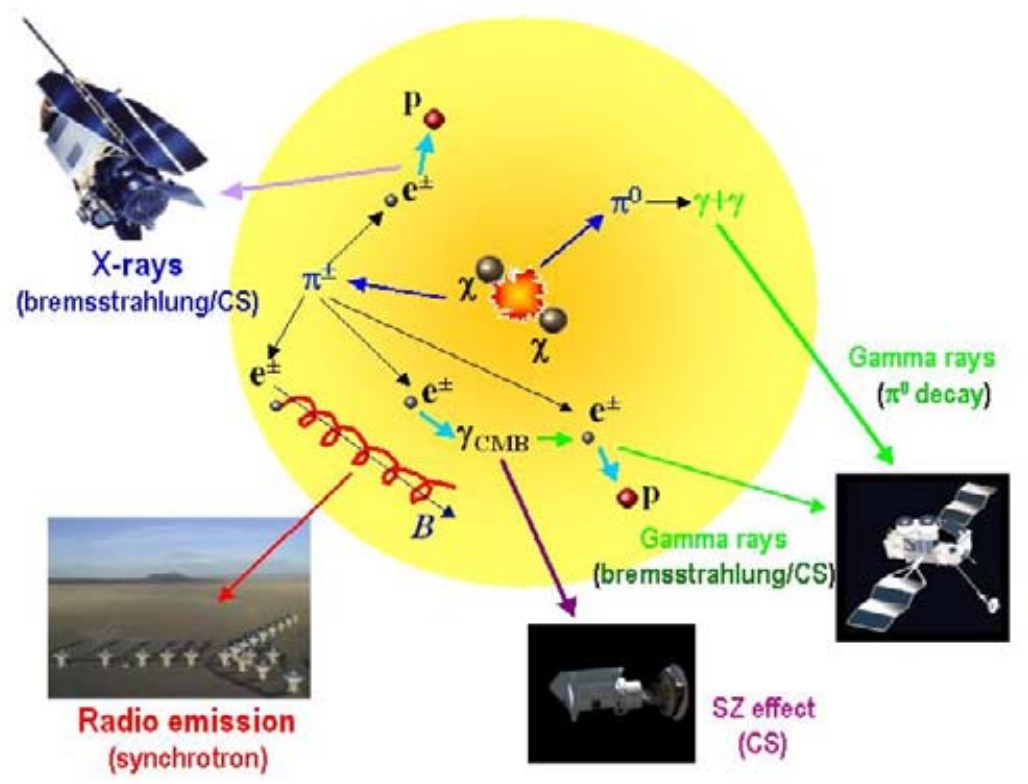

Figure 1. A simple model which shows the basic astrophysical mechanisms underlying the search for the nature of DM particles $(\chi)$ through the emission features occurring in large-scale structures (e.g., galaxy clusters and galaxies). These astrophysical mechanisms are, among others: gamma-ray emission from $\pi^{0} \rightarrow \gamma+\gamma$, relativistic bremsstrahlung of secondary electrons and CS of CMB photons by secondary electrons; X-ray/UV emission due to non-thermal bremsstrahlung and CS of background photons by secondary electrons; synchrotron emission by secondary electrons diffusing in the intra-cluster magnetic field; $\mathrm{SZ}_{D M}$ (CS of CMB photons by secondary electrons) effect. Figure from Colafrancesco (2003).

The time evolution of the secondary electron spectrum in a DM halo is described by the transport equation:

$$
\frac{\partial n_{e}(E, r)}{\partial t}-\operatorname{div}\left[D \nabla n_{e}(E, r)\right]-\frac{\partial}{\partial E}\left[n_{e}(E, r) b(E)\right]=Q_{e}(E, r)
$$

where $n_{e}(E, r)$ is the equilibrium spectrum at distance $r$ from the DM halo center for the electrons with energy $E$. The source spectrum, $Q_{e}(E, r)$, reaches its equilibrium configuration mainly due to the diffusion process and to energy losses (mainly synchrotron and Compton Scattering losses at energies $E \gtrsim 150 \mathrm{MeV}$ and to Coulomb losses at smaller energies). Since DM annihilation produces a source spectrum $Q_{e}(E, r)$ which is constant in time, the population of high-energy electrons can be described by a quasi-stationary $\left(\partial n_{e} / \partial t \approx 0\right)$ transport equation, from which the equilibrium spectrum $n_{e}(E, r)$ can be derived (see Colafrancesco, Profumo \& Ullio (2005) for details). The following qualitative solution (Colafrancesco $(2005 b)$ ) for the average electron density

$$
n_{e}(E, r) \approx\left[Q_{e}(E, r) \tau_{\text {loss }}\right] \times \frac{V_{s}}{V_{s}+V_{o}} \times \frac{\tau_{D}}{\tau_{D}+\tau_{\text {loss }}}
$$

resumes the relevant aspects of the transport eq.(2.2). Here, $V_{s} \propto R_{h}^{3}$ and $V_{o} \propto \lambda^{3}(E)$ are the volumes occupied by the DM source (we assume a spherical dwarf galaxy with radius $R_{h}$ ) and the one occupied by the diffusing electrons which travel a distance $\lambda(E) \approx$ $\left[D(E) \cdot \tau_{\text {loss }}(E)\right]^{1 / 2}$ before loosing much of their initial energy. The relevant time scales in eq.(2.2) are the diffusion time-scale, $\tau_{D} \approx R_{h}^{2} / D(E)$, and the energy loss time-scale $\tau_{\text {loss }}=E / b_{e}(E)$. The diffusion coefficient $D(E)=D_{0}\left(E / E_{0}\right)^{\gamma} B^{-\gamma}$ is assumed to be 

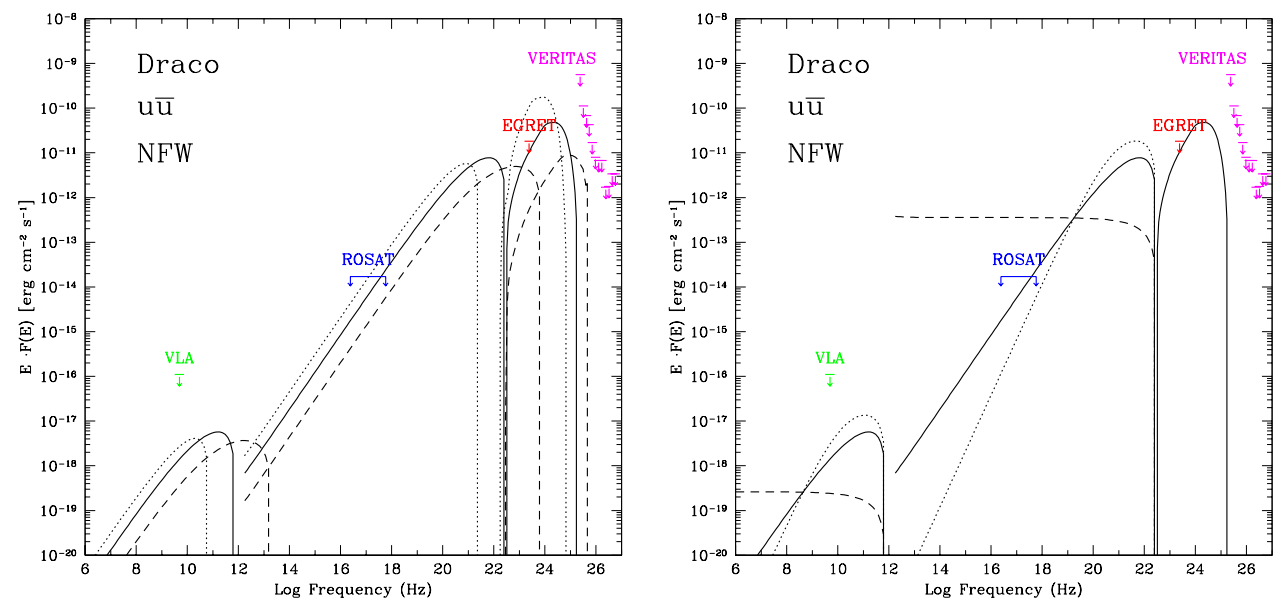

Figure 2. Left. The SED of Draco as produced by $\chi \chi$ annihilation for masses $M_{\chi}=50 \mathrm{GeV}$ (dotted), $100 \mathrm{GeV}$ (solid) and $500 \mathrm{GeV}$ (dashed). The three sets of curves refer (from low to high frequencies) to synchrotron (we assume a uniform magnetic field $B_{\mu}=3$ ), ICS and $\gamma$-rays from $\pi^{0} \rightarrow \gamma \gamma$ decay). A diffusion coefficient $D \sim E^{\gamma}$ with $\gamma=1 / 3$ is assumed here. Right. We show here the effect of a different energy dependence of the diffusion coefficient on the Draco SED produced from $\chi \chi$ annihilation: $\gamma=0$ (dotted), $\gamma=1 / 3$ (solid), $\gamma=1$ (dashed).

only dependent on the electron energy and on the galaxy magnetic field $B_{\mu}$ here given in $\mu \mathrm{G}$. The function $b_{e}(E)$ gives the electron energy loss per unit time at energy $E$ : $b_{e}(E)=b_{0}\left(B_{\mu}\right)(E / G e V)^{2}+b_{C o u l}$, where $b_{0}\left(B_{\mu}\right) \approx\left(2.5 \times 10^{-17}+2.54 \times 10^{-18} B_{\mu}^{2}\right)$ and $b_{\text {Coul }} \approx 7 \times 10^{-16}\left[n(r) / 1 \mathrm{~cm}^{-3}\right]$, if $b_{e}$ is given in units of $\mathrm{GeV} / \mathrm{s}$.

For $E>E_{*}=\left(D_{0} E_{0} / R_{h}^{2} b_{0} B_{\mu}^{\gamma}\right)^{1 /(1-\gamma)}$, the condition $\tau_{D}>\tau_{\text {loss }}$ (and consistently $\lambda(E)<R_{h}$ ) holds, the diffusion is not relevant and the solution of eq.(2.2) is $n_{e} \sim$ $Q_{e}(E, r) \tau_{\text {loss }}$ and shows an energy spectrum $\sim Q(E) \cdot E^{-1}$. For $E<E_{*}$, the condition $\tau_{D}<\tau_{\text {loss }}$ (and consistently $\lambda(E)>R_{h}$ ) holds, the diffusion is relevant and the solution of eq.(2.2) is $n_{e} \sim\left[Q_{e}(E, r) \tau_{D}\right] \times\left(V_{s} / V_{o}\right)$ and shows an energy spectrum $\sim Q(E) \cdot E^{(2-5 \gamma) / 2}$ which is flatter or equal to the previous case for reasonable values $\gamma=1 / 3-1$. This last situation $\left(\lambda(E)>R_{h}, \tau_{D}<\tau_{\text {loss }}\right)$ applies to the regime of dwarf galaxies and, specifically, to the case of Draco.

\subsection{Multi- $\nu$ emission from DM annihilation}

The astrophysical signals of DM annihilation can be evaluated from the equilibrium spectrum $n_{e}(E, r)$ and populate the entire e.m. spectrum, from radio-waves to gamma-rays (see Colafrancesco (2005b), Colafrancesco, Profumo \& Ullio (2005) for details). We show in Fig.2 the overall spectrum of Draco as produced by the decay and emission of secondary products of $\chi \chi$ annihilation. We briefly discuss here the main features of the overall spectral energy distribution (SED) of Draco derived from DM annihilation and we refer the interested reader to Colafrancesco (2005b) for the discussion of the technical aspects of the illumination of dwarf galaxies from DM annihilation. Gamma-ray emission is predominantly due to the hadronization of the decay products of $\chi \chi$ with the production of a continuum spectrum due to the decay $\pi^{0} \rightarrow \gamma+\gamma$ (see Colafrancesco \& Mele (2001), Colafrancesco (2005a)), even though the direct neutralino annihilation results in a line emission feature at an energy $\sim M_{\chi}$. Gamma-ray emission is the most direct signal of DM annihilation and it could be revealed provided that i) sufficient spectral and spatial 
resolution will be achieved by the next coming experiments and, ii) a clear understanding of other competing gamma-ray emission mechanisms (like cosmic-ray acceleration) expected to be at work in DM halos (see Colafrancesco (2003), Colafrancesco (2005b)) will be obtained. Gamma-ray emission is also expected from secondary electrons produced by $\chi \chi$ annihilation via $\chi \chi \rightarrow \pi^{ \pm}+X, \pi^{ \pm} \rightarrow \mu^{ \pm} \nu_{\mu}\left(\bar{\nu}_{\mu}\right), \mu^{ \pm} \rightarrow e^{ \pm}+\bar{\nu}_{\mu}\left(\nu_{\mu}\right)+\nu_{e}\left(\bar{\nu}_{e}\right)$. These secondary electrons may produce gamma-rays through bremsstrahlung and ICS of CMB photons up to high energies (see Colafrancesco (2003), Colafrancesco (2005a)). We show in Fig. 2 the $\gamma$-ray flux from ICS and $\pi^{0}$ decay predicted for Draco. The present $\gamma$-ray limits from EGRET and from VERITAS are also shown.

The ICS of CMB photons by means of secondary electrons produces a diffuse emission in the DM halo of Draco which extends in energy even down to the IR, optical, UV and X-ray frequency bands with the same spectrum (see Fig.2). An X-ray upper limit for Draco has been obtained from ROSAT PSPC observations (Zang \& Meurs (2001)) and is already able to put severe constraints on the DM particle nature, as well as on the diffusion coefficient of relativistic particles in dwarf galaxies.

The same secondary electrons produced by $\chi \chi$ annihilation unavoidably produce synchrotron emission (if a magnetized atmosphere is present) which can be observed at radio frequencies as a diffuse and extended radio-halo centered on the center of the DM halo. Observations of Draco at $4.9 \mathrm{GHz}$ (Fomalont \& Geldzhaler(1979)) only provides an upper limit.

The production of secondary electrons from DM annihilation provides other interesting and relevant effects like i) an heating of the residual gas (left in the galaxy atmosphere after the star formation phase) through Coulomb scattering (Colafrancesco (2005b) and ii) the ICS distortion of the CMB spectrum (Colafrancesco (2004a)). In this context, it has been recently proposed (Colafrancesco (2004a)) that the unavoidable ICS of CMB photons by the secondary electrons produced in $\chi \chi$ annihilation provides a source of SZ effect, that we call here $\mathrm{SZ}_{D M}$, along the line of sight through the dwarf galaxy. Such $\mathrm{SZ}_{D M}$ effect has very specific spectral and spatial features that allow its detection in future high-sensitivity CMB experiments.

\subsection{Constraints on DM properties}

The illumination of dwarf galaxies produced by DM annihilation is able to provide strong constraints to the $\chi$ physical properties. Fig. 3 shows, in fact, the constraints set by the spectral energy distribution of Draco on the $\langle\sigma V\rangle_{A}-M_{\chi}$ plane combined with the WMAP and BBN constraints. The present analysis shows that even the available upper limits for Draco are able to strongly constrain $\chi$ DM models. The main astrophysical constraint so far comes from the ICS emission of secondary electrons compared to the X-ray and SZ experimental limits.

The results of this combined analysis are only preliminary since we concentrated on $u \bar{u}$ and $W^{-} W^{+}$neutralino models (see Colafrancesco \& Profumo (2005) for details), and a more refined analysis of the exclusion plots shown in Fig.3 is being performed for several specific models of neutralinos predicted in supersymmetric theories for a wide choice of masses and physical compositions of neutralinos that can be relevant as CDM candidates (Colafrancesco, Profumo \& Ullio (2005)). Nonetheless, the results presented here are already indicative of the constraining power of astrophysical observations of DM dominated structures (like dwarf galaxies). Further restrictions of the previous exclusion plot may be obtained by comparing astrophysical constrains to those coming from both accelerator physics and from other cosmological probes which are sensitive to the amount and nature of the DM. 

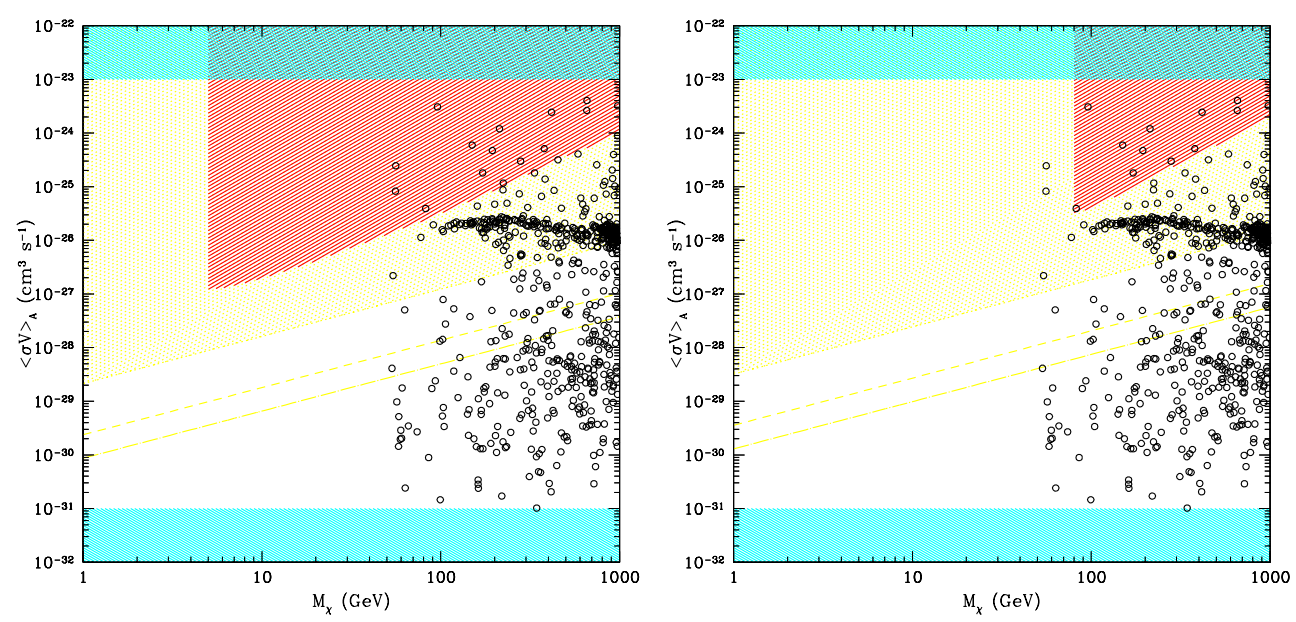

Figure 3. The exclusion regions obtained from Draco (yellow areas, a diffusion coefficient $D \propto E^{\gamma}$ with $\gamma=1$ is used here), from Big Bang Nucleosynthesis (BBN) (red areas, see Jedamzik (2004)) and from WMAP are shown for the two neutralino models here considered: the $u \bar{u}$ (left panel) and the $W^{-} W^{+}$models (right panel). The dotted circles in each panel represent specific supersymmetric models in these two classes which have relic abundance consistent with the WMAP limits (see Colafrancesco \& Profumo (2005) dor details). The dashed and dot-dashed curves represents the limits from Draco with an enhancement factor due to DM clumpiness of $f=3$ and 5 , respectively.

\section{Conclusion}

The nature of DM, despite its overwhelming evidence, is still intangible. It is nonetheless appealing, in these respects, that some astrophysical features of dwarf galaxies might give information on the fundamental properties of the DM particles.

\section{References}

Colafrancesco, S. 2003, ChJAA 3, 126

Colafrancesco, S. 2004, A\&A 422, L23

Colafrancesco, S. 2005, in: F.Giovannelli \& G. Mannocchi (eds.), Frontier Objects in Astrophysics and Particle Physics in press

Colafrancesco, S. 2005, A\& $A$ submitted

Colafrancesco, S. \& Mele, B. 2001, ApJ 562, 24

Colafrancesco, S. \& Profumo, S. 2005, A\& $A$ submitted

Colafrancesco, S., Profumo, S. \& Ullio, P. 2005, A\&A submitted

Fomalont, E. \& Geldzhaler, B. 1979, AJ 84, 12

Gondolo, P. et al. 2004, JCAP 0407, 008

Jedamzik, K. 2004, Ph.Rev.D 70, 3510

Jungman, G. et al. 1996, Phys.Rep. 267, 195

Navarro, J., Frenk, C. \& White, S.D.M. 1997, ApJ 490, 493

Page, L. 2003, preprint astro-ph/0306381

Spergel, D.N. et al. 2003, ApJS 148, 175

Zang, Z. \& Meurs, E.J.A. 2001, ApJ 556, 24

Zwicky, F. 1933, Helv.Phys.Acta 6, 110 